\title{
Nonstationary INAR(1) Process with qth-Order Autocorrelation Innovation
}

\author{
Kaizhi Yu, ${ }^{1}$ Hong Zou, ${ }^{2}$ and Daimin Shi ${ }^{1}$ \\ ${ }^{1}$ Statistics School, Southwestern University of Finance and Economics, Chengdu, Sichuan 611130, China \\ ${ }^{2}$ School of Economics, Southwestern University of Finance and Economics, Chengdu, Sichuan 611130, China
}

Correspondence should be addressed to Kaizhi Yu; kaizhiyu.swufe@gmail.com

Received 7 January 2013; Accepted 17 February 2013

Academic Editor: Fuding Xie

Copyright (c) 2013 Kaizhi Yu et al. This is an open access article distributed under the Creative Commons Attribution License, which permits unrestricted use, distribution, and reproduction in any medium, provided the original work is properly cited.

This paper is concerned with an integer-valued random walk process with $q$ th-order autocorrelation. Some limit distributions of sums about the nonstationary process are obtained. The limit distribution of conditional least squares estimators of the autoregressive coefficient in an auxiliary regression process is derived. The performance of the autoregressive coefficient estimators is assessed through the Monte Carlo simulations.

\section{Introduction}

In many practical settings, one often encounters integervalued time series, that is, counts of events or objects at consecutive points in time. Typical examples include daily large transactions of Ericsson B, monthly number of ongoing strikes in a particular industry, number of patients treated each day in an emergency department, and daily counts of new swine flu cases in Mexico. Since most traditional representations of dependence become either impossible or impractical; see Silva et al. [1], this area of research did not attract much attention until the early 1980s. During the last three decades, a number of time series models have been developed for discrete-valued data. It has become increasingly important to gain a better understanding of the probabilistic properties and to develop new statistical techniques for integer-valued time series.

The existing models can be broadly classified into two types: thinning operator models and regression models. Recently the thinning operator models have been greatly developed; see a survey by Weiß [2]. A number of innovations have been made to model various integer-valued time series. For instance, Ferland et al. [3] proposed an integer-valued GARCH model to study overdispersed counts, and Fokianos and Fried [4], Weiß [5], and Zhu and Wang [6-8] made further studies. Bu and McCabe [9] considered the lag-order selection for a class of integer autoregressive models, while Enciso-Mora et al. [10] discussed model selection for general integer-valued autoregressive moving-average processes. Silva et al. [1] addressed a forecasting problem in an INAR(1) model. McCabe et al. [11] derived efficient probabilistic forecasts of integer-valued random variables. Several other models and thinning operators were proposed as well.

Random coefficient INAR models were studied by Zheng et al. [12, 13] and Gomes and e Castro [14], while random coefficient INMA models were proposed by Yu et al. $[15,16]$. Kachour and Yao [17] introduced a class of autoregressive models for integer-valued time series using the rounding operator. Kim and Park [18] proposed an extension of integervalued autoregressive INAR models by using a signed version of the thinning operator. The signed thinning operator was developed by Zhang et al. [19] and Kachour and Truquet [20].

However, these models focus exclusively on stationary processes, whereas nonstationary processes are often encountered in reality. Some studies have examined asymptotic properties of nonstationary INAR models for nonstatinaory time series. Hellström [21] focused on the testing of unit root in INAR(1) models and provided small sample distributions for the Dickey-Fuller test statistic. Ispány et al. [22] considered a nearly nonstationary INAR(1) process. It was shown that the limiting distribution of the conditional least squares estimator for the coefficient is normal. Györfi et al. [23] 
proposed a nonstationary inhomogeneous INAR(1) process, where the autoregressive type coefficient slowly converges to one. Kim and Park [18] considered a process called integervalued autoregressive process with signed binomial thinning to handle a nonstationary integer-valued time series with a large dispersion. Drost et al. [24] studied the asymptotic properties of this "near unit root" situation. They found that the limit experiment is Poissonian. Barczy et al. [25] proved that the sequence of appropriately scaled random step functions formed from an unstable $\operatorname{INAR}(p)$ process that converges weakly towards a squared Bessel process.

However, to our knowledge, few effort has been devoted to studying nonstationary INAR(1) models with an INMA innovation. In this paper, we aim to fill in this gap in the literature. We consider a new nonstatioary INAR(1) process in which the innovation follows a $q$ th-order moving average process (NSINARMA $(1, q)$ ). Similar to the studies of nonstationary processes for continuous time series models, we need to accommodate two stochastic processes, one as the "true process" and the other as an "auxiliary regression process." In this paper, we study particularly statistical properties of the conditional least squares (CLS) estimators of the autoregressive coefficient in the auxiliary integer-valued autoregression process, when the "true process" is nonstationary integervalued autoregression with an innovation which has an integer-valued moving average part.

The rest of this paper is organized as follows. In Section 2, our nonstationary thinning model with $\operatorname{INMA}(q)$ innovation is described, and some statistical properties are established. In Section 3, the limiting distribution of the autoregressive coefficient in the auxiliary regression process is derived. In Section 4, simulation results for the CLS estimator are presented. Finally, concluding remarks are made in Section 5

\section{Definition and Properties of the NSINARMA $(1, q)$ Process}

In this section, we consider a nonstationary INAR(1) process which can be used to deal with the autocorrelation of innovation process.

Definition 1. An integer-valued stochastic process $\left\{X_{t}\right\}$ is said to be the NSINARMA $(1, q)$ process if it satisfies the following recursive equations:

$$
\begin{gathered}
X_{t}=X_{t-1}+u_{t}, \\
u_{t}=\varepsilon_{t}+\theta_{1} \circ \varepsilon_{t-1}+\theta_{2} \circ \varepsilon_{t-2}+\cdots+\theta_{q} \circ \varepsilon_{t-q},
\end{gathered}
$$

where $\left\{\varepsilon_{t}\right\}$ is a sequence of i.i.d. nonnegative integer-valued random variables with finite mean $\mu_{\varepsilon}<\infty$, variance $\sigma_{\varepsilon}^{2}<\infty$, and probability mass function $f_{\varepsilon}$. All counting series $\theta_{k} \circ \varepsilon_{t-k}$ are mutually independent, and $\theta_{k} \in[0,1], k=1, \ldots, q$. For the sake of convenience, we suppose that the process starts from zero, more precisely, $X_{0}=0$.

It is easy to see the following results of $X_{t}$ hold.
Proposition 2. For $t \geq 1$, one has

(i) $E\left(X_{t} X_{t-1}\right)=X_{t-1}+\left(1+\theta_{1}+\cdots+\theta_{q}\right) \mu_{\varepsilon}$,

(ii) $E\left(X_{t}\right)=t\left(1+\theta_{1}+\cdots+\theta_{q}\right) \mu_{\varepsilon}$,

(iii) $\operatorname{Var}\left(X_{t} X_{t-1}\right)=\mu_{\varepsilon} \sum_{k=1}^{q} \theta_{k}\left(1-\theta_{k}\right)+\sigma_{\varepsilon}^{2}\left(1+\sum_{k=1}^{q} \theta_{k}^{2}\right)$,

(iv) $\operatorname{Var}\left(X_{t}\right)=t\left(\mu_{\varepsilon} \sum_{k=1}^{q} \theta_{k}\left(1-\theta_{k}\right)+\sigma_{\varepsilon}^{2}\left(1+\sum_{k=1}^{q} \theta_{k}^{2}\right)\right)$.

Proof. It is straightforward to get (i) to (iii). We prove (iv) by induction with

$$
\begin{aligned}
\operatorname{Var} & \left(X_{t}\right) \\
& =\operatorname{Var}\left(E\left(X_{t} \mid X_{t-1}\right)\right)+E\left(\operatorname{Var}\left(X_{t} \mid X_{t-1}\right)\right) \\
& =\operatorname{Var}\left(X_{t-1}\right)+\left(\mu_{\varepsilon} \sum_{k=1}^{q} \theta_{k}\left(1-\theta_{k}\right)+\sigma_{\varepsilon}^{2}\left(1+\sum_{k=1}^{q} \theta_{k}^{2}\right)\right)
\end{aligned}
$$

and the initial value $X_{0}=0$.

\section{Estimation Methods}

Similar to the continuous time series process with unit roots, we focus on the properties of the autoregressive coefficient estimator in an auxiliary regression process when the true process follows the nonstatioary INAR(1) process defined as Definition 1.

Suppose that the auxiliary regression process $X_{t}$ is an INAR(1) model,

$$
X_{t}=\alpha \circ X_{t-1}+v_{t}
$$

where $\left\{v_{t}\right\}$ is a sequence of i.i.d. nonnegative integer-valued random variables with finite mean $\mu_{v}$ and variance $\sigma_{v}^{2}$. We are interested in the properties of $\alpha$ when the true process is a nonstatioary INAR(1) model with moving average components. In this paper, we consider a conditional least squares (CLSs) estimator. An advantage of this method is that it does not require specifying the exact family of distributions for the innovations.

Let

$$
Q(\beta)=\sum_{t=1}^{T}\left(X_{t}-\alpha \circ X_{t-1}-\mu_{v}\right)^{2},
$$

with $\beta=\left(\alpha, \mu_{v}\right)$, be the CLS criterion function. The CLS estimators of $\alpha$ and $\mu_{v}$ are obtained by minimizing $Q$ and are given by

$$
\begin{gathered}
\widehat{\alpha}=\frac{T \sum_{t=1}^{T} X_{t-1} X_{t}-\left(\sum_{t=1}^{T} X_{t-1}\right)\left(\sum_{t=1}^{T} X_{t}\right)}{T \sum_{t=1}^{T} X_{t-1}^{2}-\left(\sum_{t=1}^{T} X_{t-1}\right)^{2}}, \\
\widehat{\mu}_{v}=T^{-1}\left(\sum_{t=1}^{T} X_{t}-\widehat{\alpha} \sum_{t=1}^{T} X_{t-1}\right) .
\end{gathered}
$$

Similar to studying the unit root in continuous time series, we are only concerned with statistical properties of 
the autoregressive coefficient estimator. For the nonstationary of continuous-valued time series, we often need to examine whether the characteristic polynomial of $\mathrm{AR}(1)$ process has a unit root. Thus, we want to see if we can find the limiting distribution of the autoregressive coefficient estimator. Let us present a result that is needed later on.

Lemma 3. Suppose that $Z_{t}$ follows a random walk without drift,

$$
Z_{t}=Z_{t-1}+w_{t}
$$

where $Z_{0}=0$ and $\left\{w_{t}\right\}$ is an i.i.d. sequence with mean zero and variance $\sigma_{w}^{2}>0$. Let " $\Rightarrow$ " denote converges in distribution, and let $W(r)$ denote the standard Brownian motion. Then, one has the following properties:

(i) $T^{-1 / 2} \sum_{t=1}^{T} w_{t} \Rightarrow \sigma_{w} W(1)$,

(ii) $T^{-1} \sum_{t=1}^{T} Z_{t-1} w_{t} \Rightarrow(1 / 2) \sigma_{w}^{2}\left[W^{2}(1)-1\right]$,

(iii) $T^{-3 / 2} \sum_{t=1}^{T} t w_{t} \Rightarrow \sigma_{w} W(1)-\sigma_{w} \int_{0}^{1} W(r) d r$,

(iv) $T^{-3 / 2} \sum_{t=1}^{T} Z_{t-1} \Rightarrow \sigma_{w} \int_{0}^{1} W(r) d r$,

(v) $T^{-5 / 2} \sum_{t=1}^{T} t Z_{t-1} \Rightarrow \sigma_{w} \int_{0}^{1} r W(r) d r$,

(vi) $T^{-2} \sum_{t=1}^{T} Z_{t-1}^{2} \Rightarrow \sigma_{w}^{2} \int_{0}^{1} W^{2}(r) d r$,

(vii) $T^{-(n+1)} \sum_{t=1}^{T} t^{n} \rightarrow 1 /(n+1)$, for $n=0,1,2, \ldots$.

Proof. See Proposition 17.1 in Hamilton [26].

Theorem 4. If all the assumptions of Definition 1 hold, then one has

(i) $T^{-1} \sum_{t=1}^{T} \varepsilon_{t}\left(\theta_{k} \circ \varepsilon_{t-k}\right)$ converges in mean square to $\theta_{k} \mu_{\varepsilon}^{2}$, for $k=1, \ldots, q$,

(ii) $T^{-1} \sum_{t=1}^{T}\left(\theta_{i} \circ \varepsilon_{t-i}\right)\left(\theta_{j} \circ \varepsilon_{t-j}\right)$ converges in mean square to $\theta_{i} \theta_{j} \mu_{\varepsilon}^{2}$, for $1 \leq i<j \leq q$.

Proof. (i) First, we prove that $T^{-1} \sum_{t=1}^{T} \varepsilon_{t} \cdot\left(\theta_{k} \circ \varepsilon_{t-k}\right)$ is integrable in mean square. Using the well-known results:

$$
\begin{aligned}
E(X(\beta \circ Y)) & =E(X) E(\beta \circ Y)=\beta E(X) E(Y), \\
E(\beta \circ Y)^{2} & =E\left(E\left((\beta \circ Y)^{2} \mid Y\right)\right) \\
& =\beta(1-\beta) E(Y)+\beta^{2} E\left(Y^{2}\right),
\end{aligned}
$$

where $X$ and $Y$ are independent and $\beta \in[0,1]$, we can derive that

$$
\begin{aligned}
E\left(T^{-1} \sum_{t=1}^{T} \varepsilon_{t}\left(\theta_{k} \circ \varepsilon_{t-k}\right)\right)^{2} & \\
= & T^{-2}\left(\sum_{t=1}^{T} E\left[\varepsilon_{t}^{2}\left(\theta_{k} \circ \varepsilon_{t-k}\right)^{2}\right]\right. \\
& \left.+2 \sum_{1 \leq m<n \leq T} E\left(\varepsilon_{m} \cdot \theta_{k} \circ \varepsilon_{m-k}\right)\left(\varepsilon_{n} \cdot \theta_{k} \circ \varepsilon_{n-k}\right)\right) \\
= & T^{-2}\left(T \left(( \mu _ { \varepsilon } ^ { 2 } + \sigma _ { \varepsilon } ^ { 2 } ) \left(\theta_{k}\left(1-\theta_{k}\right) \mu_{\varepsilon}\right.\right.\right. \\
= & \left.T^{-1}\left(\left(\mu_{\varepsilon}^{2}+\sigma_{\varepsilon}^{2}\right)\left(\theta_{k}^{2}\left(1-\theta_{\varepsilon}^{2}+\sigma_{\varepsilon}^{2}\right)\right)\right)+\left(T^{2}-T\right) \theta_{k}^{2} \mu_{\varepsilon}^{4}\right) \\
& \left.+\left(1-T_{k}^{2}\left(\mu_{\varepsilon}^{2}+\sigma_{\varepsilon}^{2}\right)\right)\right) \\
\leq & \left(\left(\mu_{\varepsilon}^{2}+\sigma_{\varepsilon}^{2}\right)\left(\theta_{k}^{2} \mu_{\varepsilon}^{4}\left(1-\theta_{k}\right) \mu_{\varepsilon}+\theta_{k}^{2}\left(\mu_{\varepsilon}^{2}+\sigma_{\varepsilon}^{2}\right)\right)\right) \\
& +\theta_{k}^{2} \mu_{\varepsilon}^{4}<\infty .
\end{aligned}
$$

Therefore, $T^{-1} \sum_{t=1}^{T} \varepsilon_{t} \cdot\left(\theta_{k} \circ \varepsilon_{t-k}\right)$ is integrable.

Next, we show that it converges to $\theta_{k} \mu_{\varepsilon}^{2}$ in mean square. In fact,

$$
\begin{aligned}
E\left(T^{-1} \sum_{t=1}^{T} \varepsilon_{t} \cdot \theta_{k} \circ \varepsilon_{t-k}-\theta_{k} \mu_{\varepsilon}^{2}\right)^{2} & \\
= & E\left(T^{-1} \sum_{t=1}^{T} \varepsilon_{t} \cdot \theta_{k} \circ \varepsilon_{t-k}-T^{-1} \sum_{t=1}^{T} E\left(\varepsilon_{t} \cdot \theta_{k} \circ \varepsilon_{t-k}\right)\right)^{2} \\
= & T^{-2} \sum_{t=1}^{T}\left(E\left(\varepsilon_{t} \cdot \theta_{k} \circ \varepsilon_{t-k}\right)^{2}-\left(E\left(\varepsilon_{t} \cdot \theta_{k} \circ \varepsilon_{t-k}\right)\right)^{2}\right) \\
& +2 T^{-2} \sum_{t=k+1}^{T-k} \operatorname{cov}\left(\theta_{k} \circ \varepsilon_{t-k} \cdot \varepsilon_{t}, \theta_{k} \circ \varepsilon_{t} \cdot \varepsilon_{t+k}\right) \\
= & T^{-1}\left(( \mu _ { \varepsilon } ^ { 2 } + \sigma _ { \varepsilon } ^ { 2 } ) \left(\theta_{k}\left(1-\theta_{k}\right) \mu_{\varepsilon}\right.\right. \\
& \left.\left.+\theta_{k}^{2}\left(\mu_{\varepsilon}^{2}+\sigma_{\varepsilon}^{2}\right)\right)-\theta_{k}^{2} \mu_{\varepsilon}^{4}\right) \\
& +2 T^{-2}(T-2 k) \theta_{k}^{2} \mu_{\varepsilon}^{2} \sigma_{\varepsilon}^{2} .
\end{aligned}
$$

From the assumptions $\mu_{\varepsilon}<\infty, \sigma_{\varepsilon}^{2}<\infty$, and $\theta_{k} \in[0,1]$, we get $\lim _{T \rightarrow \infty} E\left(T^{-1} \sum_{t=1}^{T} \varepsilon_{t} \cdot\left(\theta_{k} \circ \varepsilon_{t-k}\right)-\theta_{k} \mu_{\varepsilon}^{2}\right)^{2}=0$.

This completes the proof for (i).

(ii) Without loss of generality, we assume that $1 \leq i<j \leq$ $q$. We have 


$$
\begin{aligned}
& E\left(T^{-1} \sum_{t=1}^{T}\left(\theta_{i} \circ \varepsilon_{t-i}\right) \cdot\left(\theta_{j} \circ \varepsilon_{t-j}\right)\right)^{2} \\
&=T^{-2} \sum_{t=j}^{T} E\left(\left(\theta_{i} \circ \varepsilon_{t-i}\right)\left(\theta_{j} \circ \varepsilon_{t-j}\right)\right)^{2} \\
&+2 T^{-2} \sum_{1 \leq m<n \leq T} E\left(\left(\theta_{i} \circ \varepsilon_{m-i}\right)\right. \\
&= T^{-1}\left(\theta_{i}\left(1-\theta_{i}\right) \mu_{\varepsilon}+\theta_{i}^{2}\left(\mu_{\varepsilon}^{2}+\sigma_{\varepsilon}^{2}\right)\right) \\
& \times\left(\theta_{j}\left(1-\theta_{j-j}\right)\left(\theta_{i} \circ \varepsilon_{\varepsilon-i}\right)\right. \\
&\left.+\theta_{j}^{2}\left(\mu_{\varepsilon}^{2}+\sigma_{\varepsilon}^{2}\right)\right) \\
& \leq\left(\theta_{i}\left(1-\theta_{i}\right) \mu_{\varepsilon}+\theta_{i}^{2}\left(\mu_{\varepsilon}^{2}+\sigma_{\varepsilon}^{2}\right)\right) \\
& \times\left(\theta_{j}\left(1-\theta_{j}\right) \mu_{\varepsilon}+\theta_{j}^{2}\left(\mu_{\varepsilon}^{2}+\sigma_{\varepsilon}^{2}\right)\right) \\
&+\frac{1}{2} \theta_{i}^{2} \theta_{j}^{2} \mu_{\varepsilon}^{4}<\infty .
\end{aligned}
$$

Thus, $T^{-1} \sum_{t=1}^{T}\left(\theta_{i} \circ \varepsilon_{t-i}\right) \cdot\left(\theta_{j} \circ \varepsilon_{t-j}\right)$ is integrable.

Next, we prove that the limit holds $\lim _{T \rightarrow \infty} E$ $\left(T^{-1} \sum_{t=1}^{T}\left(\theta_{i} \circ \varepsilon_{t-i}\right) \cdot\left(\theta_{j} \circ \varepsilon_{t-j}\right)-\theta_{i} \theta_{j} \mu_{\varepsilon}^{2}\right)^{2}=0$,

$$
\begin{aligned}
& E\left(T^{-1} \sum_{t=1}^{T}\left(\theta_{i} \circ \varepsilon_{t-i}\right) \cdot\left(\theta_{j} \circ \varepsilon_{t-j}\right)-\theta_{i} \theta_{j} \mu_{\varepsilon}^{2}\right)^{2} \\
& =E\left(T^{-1} \sum_{t=1}^{T}\left(\theta_{i} \circ \varepsilon_{t-i}\right) \cdot\left(\theta_{j} \circ \varepsilon_{t-j}\right)\right. \\
& \left.-T^{-1} \sum_{t=1}^{T} E\left(\left(\theta_{i} \circ \varepsilon_{t-i}\right) \cdot\left(\theta_{j} \circ \varepsilon_{t-j}\right)\right)\right)^{2} \\
& =T^{-2} \sum_{t=1}^{T} \operatorname{Var}\left(\left(\theta_{i} \circ \varepsilon_{t-i}\right)\left(\theta_{j} \circ \varepsilon_{t-j}\right)\right) \\
& +2 T^{-2} \sum_{1 \leq m<n \leq T} \operatorname{cov}\left(\left(\theta_{i} \circ \varepsilon_{m-i}\right) \cdot\left(\theta_{j} \circ \varepsilon_{m-j}\right),\right. \\
& \left.\left(\theta_{i} \circ \varepsilon_{n-i}\right) \cdot\left(\theta_{j} \circ \varepsilon_{n-j}\right)\right) \\
& =T^{-2} \sum_{t=1}^{T} \operatorname{Var}\left(\left(\theta_{i} \circ \varepsilon_{t-i}\right)\left(\theta_{j} \circ \varepsilon_{t-j}\right)\right) \\
& +T^{-2} \sum_{k=\max \{1,1-i+j\}}^{T-i+j} \operatorname{cov}\left(\left(\theta_{i} \circ \varepsilon_{k-i}\right) \cdot\left(\theta_{j} \circ \varepsilon_{k-j}\right),\right. \\
& \left.\left(\theta_{i} \circ \varepsilon_{k-2 i+j}\right) \cdot\left(\theta_{j} \circ \varepsilon_{k-i}\right)\right)
\end{aligned}
$$

$$
\begin{aligned}
&= T^{-1}\left(\theta_{i}\left(1-\theta_{i}\right) \mu_{\varepsilon}+\theta_{i}^{2}\left(\mu_{\varepsilon}^{2}+\sigma_{\varepsilon}^{2}\right)\right) \\
& \times\left(\theta_{j}\left(1-\theta_{j}\right) \mu_{\varepsilon}+\theta_{j}^{2}\left(\mu_{\varepsilon}^{2}+\sigma_{\varepsilon}^{2}\right)\right) \\
&+T^{-2}((T-i+j-\max \{1,1-i+j\}) \\
&\left.\quad \times \theta_{i}^{2} \theta_{j}\left(1-\theta_{j}\right) \mu_{\varepsilon}^{3}+\theta_{i}^{2} \theta_{j}^{2} \mu_{\varepsilon}^{2} \sigma_{\varepsilon}^{2}\right) .
\end{aligned}
$$

By using $\mu_{\varepsilon}<\infty, \sigma_{\varepsilon}^{2}<\infty$, and $\theta_{k} \in[0,1]$, with the above arguments, we get

$$
\lim _{T \rightarrow \infty} E\left(T^{-1} \sum_{t=1}^{T}\left(\theta_{i} \circ \varepsilon_{t-i}\right)\left(\theta_{j} \circ \varepsilon_{t-j}\right)-\theta_{i} \theta_{j} \mu_{\varepsilon}^{2}\right)^{2}=0 .
$$

Then, $T^{-1} \sum_{t=1}^{T}\left(\theta_{i} \circ \varepsilon_{t-i}\right)\left(\theta_{j} \circ \varepsilon_{t-j}\right)$ converges in mean square to $\theta_{i} \theta_{j} \mu_{\varepsilon}^{2}$.

Theorem 5. If the process $X_{t}$ is defined as in Definition 1, then one has

(i) $T^{-1} \sum_{t=1}^{T} u_{t} \Rightarrow \mu_{\varepsilon}\left(1+\sum_{k=1}^{q} \theta_{k}\right)$,

(ii) $T^{-2} \sum_{t=1}^{T} X_{t-1} u_{t} \Rightarrow(1 / 2) \mu_{\varepsilon}^{2}\left(1+\sum_{k=1}^{q} \theta_{k}\right)^{2}$,

(iii) $T^{-2} \sum_{t=1}^{T} t u_{t} \Rightarrow(1 / 2) \mu_{\varepsilon}\left(1+\sum_{k=1}^{q} \theta_{k}\right)$,

(iv) $T^{-2} \sum_{t=1}^{T} X_{t-1} \Rightarrow(1 / 2) \mu_{\varepsilon}\left(1+\sum_{k=1}^{q} \theta_{k}\right)$,

(v) $T^{-3} \sum_{t=1}^{T} t X_{t-1} \Rightarrow(1 / 3) \mu_{\varepsilon}\left(1+\sum_{k=1}^{q} \theta_{k}\right)$,

(vi) $T^{-3} \sum_{t=1}^{T} X_{t-1}^{2} \Rightarrow(1 / 3) \mu_{\varepsilon}^{2}\left(1+\sum_{k=1}^{q} \theta_{k}\right)^{2}$.

Proof. Let $\varepsilon_{t}^{*}=\varepsilon_{t}-\mu_{\varepsilon}, \varepsilon_{k, t}^{*}=\theta_{k} \circ \varepsilon_{t-k}-\theta_{k} \mu_{\varepsilon}, k=1, \ldots, q$. by

Then, we have the means and variances of $\varepsilon_{t}^{*}$ and $\varepsilon_{k, t}^{*}$ given

$$
\begin{gathered}
E\left(\varepsilon_{t}^{*}\right)=0, \quad \sigma_{\varepsilon_{t}^{*}}=\sqrt{\operatorname{Var}\left(\varepsilon_{t}^{*}\right)}=\sigma_{\varepsilon}, \\
E\left(\varepsilon_{k, t}^{*}\right)=0, \quad \sigma_{\varepsilon_{k, t}^{*}}=\sqrt{\operatorname{Var}\left(\varepsilon_{k, t}^{*}\right)}=\sqrt{\theta_{k}\left(1-\theta_{k}\right) \mu_{\varepsilon}+\theta_{k}^{2} \sigma_{\varepsilon}^{2}} .
\end{gathered}
$$

It is easy to see that $\left\{\varepsilon_{t}^{*}\right\}$ is a sequence of i.i.d. random variables. For a fixed $k,\left\{\varepsilon_{k, t}^{*}\right\}$ is also a sequence of i.i.d. random variables.

(i) Straightforward using the law of large numbers.

(ii) One has

$$
\begin{aligned}
\sum_{t=1}^{T} X_{t-1} u_{t}= & X_{0} u_{1}+X_{1} u_{2}+\cdots+X_{T-1} u_{T} \\
= & 0+u_{1} u_{2}+\left(u_{1}+u_{2}\right) u_{3} \\
& +\cdots+\left(u_{1}+\cdots+u_{T-1}\right) u_{T} \\
= & u_{1}\left(u_{2}+\cdots+u_{T}\right)+u_{2}\left(u_{3}+\cdots+u_{T}\right) \\
& +\cdots+u_{T-1} u_{T} \\
= & \sum_{i<j} u_{i} u_{j}=\frac{1}{2}\left(\left(\sum_{t=1}^{T} u_{t}\right)^{2}-\sum_{t=1}^{T} u_{t}^{2}\right) .
\end{aligned}
$$


From the conclusion in (i), we get

$$
\left(T^{-1} \sum_{t=1}^{T} u_{t}\right)^{2} \Longrightarrow \mu_{\varepsilon}^{2}\left(1+\sum_{k=1}^{q} \theta_{k}\right)^{2}
$$

Note that

$$
\begin{aligned}
\sum_{t=1}^{T} u_{t}^{2}= & \sum_{t=1}^{T} \varepsilon_{t}^{2}+\sum_{k=1}^{q}\left(\sum_{t=1}^{T}\left(\theta_{k} \circ \varepsilon_{t-k}\right)^{2}\right) \mu_{X} \\
& +\sum_{k=1}^{q}\left(\sum_{t=1}^{T} \varepsilon_{t} \cdot\left(\theta_{k} \circ \varepsilon_{t-k}\right)\right) \\
& +2 \sum_{1 \leq i<j \leq q}\left(\sum_{t=1}^{T}\left(\theta_{i} \circ \varepsilon_{t-i}\right) \cdot\left(\theta_{j} \circ \varepsilon_{t-j}\right)\right) .
\end{aligned}
$$

Using the law of large numbers, we obtain

$$
\begin{aligned}
T^{-1} \sum_{t=1}^{T} \varepsilon_{t}^{2} & \Longrightarrow E\left(\varepsilon_{t}^{2}\right)=\left(\mu_{\varepsilon}^{2}+\sigma_{\varepsilon}^{2}\right) \\
T^{-1} \sum_{t=1}^{T}\left(\theta_{k} \circ \varepsilon_{t-k}\right)^{2} & \Longrightarrow E\left(\theta_{k} \circ \varepsilon_{t-k}\right)^{2} \\
& =\theta_{k}\left(1-\theta_{k}\right) \mu_{\varepsilon}+\theta_{k}^{2}\left(\mu_{\varepsilon}^{2}+\sigma_{\varepsilon}^{2}\right) \\
& =\sigma_{\varepsilon_{k, t}^{*}}^{2}, \quad k=1, \ldots, q .
\end{aligned}
$$

Recall Theorem 4, where $T^{-1} \sum_{t=1}^{T} \varepsilon_{t}\left(\theta_{k} \circ \varepsilon_{t-k}\right)$ converges in mean square to $\theta_{k} \mu_{\varepsilon}^{2}$ and $T^{-1} \sum_{t=1}^{T}\left(\theta_{i} \circ \varepsilon_{t-i}\right)\left(\theta_{j} \circ \varepsilon_{t-j}\right)$ converges in mean square to $\theta_{i} \theta_{j} \mu_{\varepsilon}^{2}$, and thus

$$
\begin{gathered}
T^{-1} \sum_{t=1}^{T} \varepsilon_{t} \cdot\left(\theta_{k} \circ \varepsilon_{t-k}\right) \Longrightarrow \theta_{k} \mu_{\varepsilon}^{2}, \\
T^{-1} \sum_{t=1}^{T}\left(\theta_{i} \circ \varepsilon_{t-i}\right)\left(\theta_{j} \circ \varepsilon_{t-j}\right) \Longrightarrow \theta_{i} \theta_{j} \mu_{\varepsilon}^{2} .
\end{gathered}
$$

Then, we get $T^{-1} \sum_{t=1}^{T} u_{t}^{2} \Rightarrow\left(\mu_{\varepsilon}^{2}+\sigma_{\varepsilon}^{2}\right)+\sum_{k=1}^{q} \sigma_{\varepsilon_{k, t}^{*}}^{2}+$ $2\left(\sum_{k=1}^{q} \theta_{k}+\sum_{1 \leq i<j \leq q} \theta_{i} \theta_{j}\right) \mu_{\varepsilon}^{2}$.

Therefore,

$$
\begin{aligned}
T^{-2} \sum_{t=1}^{T} X_{t-1} u_{t} & =\frac{1}{2}\left(\left(T^{-1} \sum_{t=1}^{T} u_{t}\right)^{2}-T^{-1}\left(T^{-1} \sum_{t=1}^{T} u_{t}^{2}\right)\right) \\
& \Longrightarrow \frac{1}{2}\left(\mu_{\varepsilon}^{2}\left(1+\sum_{k=1}^{q} \theta_{k}\right)^{2}-0\right) \\
& =\frac{1}{2} \mu_{\varepsilon}^{2}\left(1+\sum_{k=1}^{q} \theta_{k}\right)^{2} .
\end{aligned}
$$

(iii) Morever,

$$
\begin{aligned}
T^{-2} \sum_{t=1}^{T} t u_{t}= & T^{-2} \sum_{t=1}^{T} t\left(\varepsilon_{t}+\theta_{1} \circ \varepsilon_{t-1}+\cdots+\theta_{q} \circ \varepsilon_{t-q}\right) \\
= & T^{-2} \sum_{t=1}^{T} t\left(\varepsilon_{t}^{*}+\mu_{\varepsilon}\right)+\sum_{k=1}^{q}\left(T^{-2} \sum_{t=1}^{T} t\left(\varepsilon_{k, t}^{*}+\theta_{k} \mu_{\varepsilon}\right)\right) \\
= & T^{-1 / 2}\left(T^{-3 / 2} \sum_{t=1}^{T} t \varepsilon_{t}^{*}+\sum_{k=1}^{q}\left(T^{-3 / 2} \sum_{t=1}^{T} t \varepsilon_{k, t}^{*}\right)\right) \\
& +\mu_{\varepsilon}\left(1+\sum_{k=1}^{q} \theta_{k}\right)\left(T^{-2} \sum_{t=1}^{T} t\right) .
\end{aligned}
$$

From (iii) and (vii) of Lemma 3, we have

$$
\begin{gathered}
T^{-3 / 2} \sum_{t=1}^{T} t \varepsilon_{t}^{*} \Longrightarrow \sigma_{\varepsilon_{t}^{*}} W(1)-\sigma_{\varepsilon_{t}^{*}} \int_{0}^{1} W(r) d r, \\
T^{-3 / 2} \sum_{t=1}^{T} t \varepsilon_{k, t}^{*} \Longrightarrow \sigma_{\varepsilon_{k, t}^{*}} W(1)-\sigma_{\varepsilon_{k, t}^{*}} \int_{0}^{1} W(r) d r, \\
k=1, \ldots, q, \\
T^{-2} \sum_{t=1}^{T} t \longrightarrow \frac{1}{2} .
\end{gathered}
$$

Therefore, $T^{-2} \sum_{t=1}^{T} t u_{t} \Rightarrow 0+0+(1 / 2) \mu_{\varepsilon}\left(1+\sum_{k=1}^{q} \theta_{k}\right)=$ $(1 / 2) \mu_{\varepsilon}\left(1+\sum_{k=1}^{q} \theta_{k}\right)$. Consider the following:

(iv)

$$
\begin{aligned}
T^{-2} \sum_{t=1}^{T} X_{t-1}= & T^{-2} \sum_{t=1}^{T-1}(T-t) u_{t} \\
= & T^{-2} \sum_{t=1}^{T-1}(T-t)\left(\varepsilon_{t}+\theta_{1} \circ \varepsilon_{t-1}+\cdots+\theta_{q} \circ \varepsilon_{t-q}\right) \\
= & T^{-2} \sum_{t=1}^{T}(T-t)\left(\varepsilon_{t}^{*}+\mu_{\varepsilon}\right) \\
& +T^{-2} \sum_{k=1}^{q} \sum_{t=1}^{T}(T-t)\left(\varepsilon_{k, t}^{*}+\theta_{k} \mu_{\varepsilon}\right) \\
= & T^{-2} \sum_{t=1}^{T}(T-t) \varepsilon_{t}^{*}+\sum_{k=1}^{q}\left(T^{-2} \sum_{t=1}^{T}(T-t) \varepsilon_{k, t}^{*}\right) \\
& +\mu_{\varepsilon}\left(1+\sum_{k=1}^{q} \theta_{k}\right)\left(T^{-2} \sum_{t=1}^{T}(T-t)\right)
\end{aligned}
$$




$$
\begin{aligned}
= & T^{-1} \sum_{t=1}^{T} \varepsilon_{t}^{*}-T^{-1 / 2}\left(T^{-3 / 2} \sum_{t=1}^{T} t \varepsilon_{t}^{*}\right) \\
& +\sum_{k=1}^{q}\left(T^{-1} \sum_{t=1}^{T} \varepsilon_{k, t}^{*}-T^{-1 / 2}\left(T^{-3 / 2} \sum_{t=1}^{T} t \varepsilon_{k, t}^{*}\right)\right) \\
& +\mu_{\varepsilon}\left(1+\sum_{k=1}^{q} \theta_{k}\right)\left(T^{-2} \sum_{t=1}^{T}(T-t)\right) .
\end{aligned}
$$

By the law of large numbers, we have

$$
\begin{aligned}
& T^{-1} \sum_{t=1}^{T} \varepsilon_{t}^{*} \Longrightarrow 0, \\
& T^{-1} \sum_{t=1}^{T} \varepsilon_{k, t}^{*} \Longrightarrow 0, \quad k=1, \ldots, q .
\end{aligned}
$$

From the (iii) and (vii) of Lemma 3, we get

$$
\begin{gathered}
T^{-3 / 2} \sum_{t=1}^{T} t \varepsilon_{t}^{*} \Longrightarrow \sigma_{\varepsilon_{t}^{*}} W(1)-\sigma_{\varepsilon_{t}^{*}} \int_{0}^{1} W(r) d r, \\
T^{-3 / 2} \sum_{t=1}^{T} t \varepsilon_{k, t}^{*} \Longrightarrow \sigma_{\varepsilon_{k, t}^{*}} W(1)-\sigma_{\varepsilon_{k, t}^{*}} \int_{0}^{1} W(r) d r, \\
k=1, \ldots, q, \\
T^{-2} \sum_{t=1}^{T}(T-t) \longrightarrow \frac{1}{2} .
\end{gathered}
$$

Then, we have

$$
\begin{aligned}
T^{-2} \sum_{t=1}^{T} X_{t-1} & \Longrightarrow 0+0+0+\frac{1}{2} \mu_{\varepsilon}\left(1+\sum_{k=1}^{q} \theta_{k}\right) \\
& =\frac{1}{2} \mu_{\varepsilon}\left(1+\sum_{k=1}^{q} \theta_{k}\right) .
\end{aligned}
$$

(v) Elementary algebra gives us that

$$
\begin{aligned}
X_{t} & =\sum_{k=1}^{t} u_{k}=\sum_{k=1}^{t}\left(\varepsilon_{k}+\theta_{1} \circ \varepsilon_{k-1}+\cdots+\theta_{q} \circ \varepsilon_{k-q}\right) \\
& =\sum_{k=1}^{t} \varepsilon_{k}^{*}+\sum_{k=1}^{t} \varepsilon_{1, k}^{*}+\cdots+\sum_{k=1}^{t} \varepsilon_{q, k}^{*}+\left(1+\sum_{k=1}^{q} \theta_{k}\right) \mu_{\varepsilon} t \\
& =\xi_{t}+\sum_{i=1}^{q} \eta_{i, t}+\left(1+\sum_{k=1}^{q} \theta_{k}\right) \mu_{\varepsilon} t
\end{aligned}
$$

where $\xi_{t}=\sum_{k=1}^{t} \varepsilon_{k}^{*}, \eta_{i, t}=\sum_{k=1}^{t} \varepsilon_{i, k}^{*}, i=1, \ldots, q$ and, as assumed, $\xi_{0}=\eta_{1,0}=\cdots=\eta_{q, 0}=0$. It is easy to see that $\xi_{t}$ and $\eta_{i, t}, i=1, \ldots, q$, follow a random walk process without drift. Using (v) and (vii) of Lemma 3, we get

$$
\begin{gathered}
T^{-5 / 2} \sum_{t=1}^{T} t \xi_{t-1} \Longrightarrow \sigma_{\varepsilon_{t}^{*}} \int_{0}^{1} r W(r) d r \\
T^{-5 / 2} \sum_{t=1}^{T} t \eta_{i, t-1} \Longrightarrow \sigma_{\varepsilon_{i, t}^{*}} \int_{0}^{1} r W(r) d r, \quad i=1, \ldots, q, \\
T^{-3} \sum_{t=1}^{T} t(t-1) \longrightarrow \frac{1}{3} .
\end{gathered}
$$

Then, we get

$$
\begin{aligned}
T^{-3} \sum_{t=1}^{T} t X_{t-1}= & T^{-3} \sum_{t=1}^{T} t \xi_{t-1}+\sum_{i=1}^{q}\left(T^{-3} \sum_{t=1}^{T} t \eta_{i, t-1}\right) \\
& +\left(1+\sum_{k=1}^{q} \theta_{k}\right) \mu_{\varepsilon}\left(T^{-3} \sum_{t=1}^{T} t(t-1)\right) \\
= & T^{-1 / 2}\left(T^{-5 / 2} \sum_{t=1}^{T} t \xi_{t-1}\right) \\
& +T^{-1 / 2} \sum_{i=1}^{q}\left(T^{-5 / 2} \sum_{t=1}^{T} t \eta_{i, t-1}\right) \\
& +\left(1+\sum_{k=1}^{q} \theta_{k}\right) \mu_{\varepsilon}\left(T^{-3} \sum_{t=1}^{T} t(t-1)\right) \\
\Longrightarrow & 0+0+\frac{1}{3}\left(1+\sum_{k=1}^{q} \theta_{k} \mu_{\varepsilon}\right. \\
= & \frac{1}{3}\left(1+\sum_{k=1}^{q} \theta_{k}\right) \mu_{\varepsilon} .
\end{aligned}
$$

(vi) One has

$$
\begin{aligned}
T^{-3} \sum_{t=1}^{T} X_{t-1}^{2} \\
=T^{-3} \sum_{t=1}^{T}\left(\xi_{t-1}+\sum_{i=1}^{q} \eta_{i, t-1}+\left(1+\sum_{k=1}^{q} \theta_{k}\right) \mu_{\varepsilon}(t-1)\right)^{2} \\
=T^{-3} \sum_{t=1}^{T} \xi_{t-1}^{2}+T^{-3} \sum_{i=1}^{q} \sum_{t=1}^{T} \eta_{i, t-1}^{2} \\
\quad+\left(1+\sum_{k=1}^{q} \theta_{k}\right)^{2} \mu_{\varepsilon}^{2}\left(T^{-3} \sum_{t=1}^{T}(t-1)^{2}\right) \\
\left.\quad+2 \sum_{i=1}^{q}\left(T^{-3} \sum_{t=1}^{T} \xi_{t-1} \eta_{i, t-1}\right)^{q}{ }^{q}\right) \mu_{\varepsilon}\left(T^{-3} \sum_{t=1}^{T}\left(\xi_{t-1}+\sum_{i=1}^{q} \eta_{i, t-1}\right)(t-1)\right)
\end{aligned}
$$


Firstly, we prove $T^{-3} \sum_{t=1}^{T}\left(\xi_{t-1}+\sum_{i=1}^{q} \eta_{i, t-1}\right)(t-1) \Rightarrow 0$. By (iii) and (v) of Lemma 3, we have

$$
\begin{gathered}
T^{-3 / 2} \sum_{t=1}^{T} t \varepsilon_{t}^{*} \Longrightarrow \sigma_{\varepsilon_{t}^{*}} W(1)-\sigma_{\varepsilon_{t}^{*}} \int_{0}^{1} W(r) d r, \\
T^{-5 / 2} \sum_{t=1}^{T} t \xi_{t-1} \Longrightarrow \sigma_{\varepsilon_{t}^{*}} \int_{0}^{1} r W(r) d r .
\end{gathered}
$$

It is easy to see that $T^{-2} \xi_{T-1} \Rightarrow 0$ and $T^{-2} \varepsilon_{T}^{*} \Rightarrow 0$. Thus,

$$
\begin{aligned}
T^{-3} \sum_{t=1}^{T}(t-1) \xi_{t-1} \\
=T^{-3} \sum_{t=1}^{T-1} t \xi_{t}=T^{-3} \sum_{t=1}^{T-1} t\left(\xi_{t-1}+\varepsilon_{t}^{*}\right) \\
=T^{-3} \sum_{t=1}^{T} t\left(\xi_{t-1}+\varepsilon_{t}^{*}\right)-T^{-2} \xi_{T-1}-T^{-2} \varepsilon_{T}^{*} \\
=T^{-1 / 2}\left(T^{-5 / 2} \sum_{t=1}^{T-1} t \xi_{t-1}\right)+T^{-3 / 2}\left(T^{-3 / 2} \sum_{t=1}^{T-1} t \varepsilon_{t}^{*}\right) \\
\quad-T^{-2} \xi_{T-1}-T^{-2} \varepsilon_{T}^{*} \Longrightarrow 0 .
\end{aligned}
$$

By using a similar approach, we get $T^{-3} \sum_{t=1}^{T}(t-1) \eta_{i, t-1} \Rightarrow$ $0, i=1, \ldots, q$.

Therefore,

$$
\begin{aligned}
T^{-3} \sum_{t=1}^{T}\left(\xi_{t-1}+\sum_{i=1}^{q} \eta_{i, t-1}\right)(t-1) \\
=T^{-3} \sum_{t=1}^{T}(t-1) \xi_{t-1} \\
\quad+\sum_{i=1}^{q}\left(T^{-3} \sum_{t=1}^{T}(t-1) \eta_{i, t-1}\right) \Longrightarrow 0+0=0 .
\end{aligned}
$$

Secondly, we prove that the limit $T^{-3} \sum_{t=1}^{T} \xi_{t-1} \eta_{i, t-1} \Rightarrow$ $0, i=1, \ldots, q$, holds.

Using the well-known inequality $\left|\xi_{t-1} \eta_{i, t-1}\right| \leq(1 / 2)\left(\xi_{t-1}^{2}+\right.$ $\left.\eta_{i, t-1}^{2}\right)$, we find that

$$
\begin{aligned}
\left|T^{-3} \sum_{t=1}^{T} \xi_{t-1} \eta_{i, t-1}\right| & \leq T^{-3} \sum_{t=1}^{T}\left|\xi_{t-1} \eta_{i, t-1}\right| \\
& \leq \frac{1}{2}\left(T^{-3} \sum_{t=1}^{T} \xi_{t-1}^{2}+T^{-3} \sum_{t=1}^{T} \eta_{i, t-1}^{2}\right) .
\end{aligned}
$$

From (vi) of Lemma 3, we get

$$
\begin{aligned}
T^{-2} \sum_{t=1}^{T} \xi_{t-1}^{2} & \Longrightarrow \sigma_{\varepsilon_{t}^{*}}^{2} \int_{0}^{1} W^{2}(r) d r, \\
T^{-2} \sum_{t=1}^{T} \eta_{i, t-1}^{2} & \Longrightarrow \sigma_{\varepsilon_{i, t}^{*}}^{2} \int_{0}^{1} W^{2}(r) d r .
\end{aligned}
$$

The two limits imply that

$$
T^{-3} \sum_{t=1}^{T} \xi_{t-1}^{2} \Longrightarrow 0, \quad T^{-3} \sum_{t=1}^{T} \eta_{i, t-1}^{2} \Longrightarrow 0
$$

By the Cauchy-Schwarz theorem, we obtain $T^{-3} \sum_{t=1}^{T} \xi_{t-1}$ $\eta_{i, t-1} \Rightarrow 0, i=1, \ldots, q$.

From (iii), (vi), and (vii) of Lemma 3, we obtain

$$
\begin{gathered}
T^{-2} \sum_{t=1}^{T} \xi_{t-1}^{2} \Longrightarrow \sigma_{\varepsilon_{t}^{*}}^{2} \int_{0}^{1} W^{2}(r) d r \\
T^{-2} \sum_{t=1}^{T} \eta_{i, t-1}^{2} \Longrightarrow \sigma_{\varepsilon_{i, t}^{*}}^{2} \int_{0}^{1} W^{2}(r) d r \\
T^{-3} \sum_{t=1}^{T}(t-1)^{2} \longrightarrow \frac{1}{3} \\
T^{-3 / 2} \sum_{t=1}^{T} t \varepsilon_{t}^{*} \Longrightarrow \sigma_{\varepsilon_{t}^{*}} W(1)-\sigma_{\varepsilon_{t}^{*}} \int_{0}^{1} W(r) d r, \\
T^{-3 / 2} \sum_{t=1}^{T} t \varepsilon_{i, t}^{*} \Longrightarrow \sigma_{\varepsilon_{i, t}^{*}} W(1)-\sigma_{\varepsilon_{i, t}^{*}} \int_{0}^{1} W(r) d r .
\end{gathered}
$$

Therefore, $T^{-3} \sum_{t=1}^{T} X_{t-1}^{2} \Rightarrow 0+0+(1 / 3)\left(1+\sum_{k=1}^{q} \theta_{k}\right)^{2} \mu_{\varepsilon}^{2}+$ $0+0=(1 / 3)\left(1+\sum_{k=1}^{q} \theta_{k}\right)^{2} \mu_{\varepsilon}^{2}$.

The proof of this theorem is complete.

Theorem 6. The conditional least squares estimators of $\alpha$ given by (5) converges in distribution to constant 1 , when the true process is a nonstationary INAR(1) model with INMA(q) innovation.

Proof. We first derive the numerator limit of (5):

$$
\begin{aligned}
T^{-4}\left(T \sum_{t=1}^{T} X_{t-1} X_{t}-\left(\sum_{t=1}^{T} X_{t-1}\right)\left(\sum_{t=1}^{T} X_{t}\right)\right) \\
=T^{-3} \sum_{t=1}^{T} X_{t-1} X_{t}-\left(T^{-2} \sum_{t=1}^{T} X_{t-1}\right)\left(T^{-2} \sum_{t=1}^{T} X_{t}\right) \\
=T^{-3} \sum_{t=1}^{T} X_{t-1}^{2}+T^{-1}\left(T^{-2} \sum_{t=1}^{T} X_{t-1} u_{t}\right) \\
-\left(T^{-2} \sum_{t=1}^{T} X_{t-1}\right)\left(T^{-2} \sum_{t=1}^{T} X_{t}\right) .
\end{aligned}
$$


TABLE 1: Bias and MSE results of $\alpha$ for NSINARMA(1,1) model.

\begin{tabular}{|c|c|c|c|c|c|c|}
\hline \multicolumn{7}{|c|}{ CLS } \\
\hline \multirow[b]{2}{*}{ Sample size } & \multicolumn{3}{|c|}{$\lambda=0.3$} & \multicolumn{3}{|c|}{$\lambda=5$} \\
\hline & 100 & 300 & 800 & 100 & 300 & 800 \\
\hline \multicolumn{7}{|l|}{$\theta_{1}=0.1$} \\
\hline $\operatorname{Bias}(\alpha)$ & $-2.3411 e-03$ & $-1.3253 e-04$ & $-2.4503 e-05$ & $-9.7709 e-05$ & $1.2879 e-05$ & $9.7185 e-07$ \\
\hline $\operatorname{MSE}(\alpha)$ & $1.6443 e-03$ & $5.2694 e-06$ & $1.8012 e-07$ & $2.8641 e-06$ & $4.9761 e-08$ & $2.8335 e-10$ \\
\hline \multicolumn{7}{|l|}{$\theta_{1}=0.4$} \\
\hline $\operatorname{Bias}(\alpha)$ & $-1.8837 e-03$ & $-6.2751 e-05$ & $-1.9073 e-05$ & $-9.3204 e-05$ & $1.1088 e-05$ & $2.1430 e-06$ \\
\hline $\operatorname{MSE}(\alpha)$ & $1.0645 e-03$ & $1.1813 e-06$ & $1.0913 e-07$ & $2.6061 e-06$ & $3.6884 e-08$ & $1.3778 e-09$ \\
\hline \multicolumn{7}{|l|}{$\theta_{1}=0.7$} \\
\hline $\operatorname{Bias}(\alpha)$ & $-1.3353 e-03$ & $-8.5133 e-05$ & $-1.3719 e-05$ & $-7.6709 e-05$ & $2.1019 e-05$ & $2.5092 e-06$ \\
\hline $\operatorname{MSE}(\alpha)$ & $5.3488 e-04$ & $2.1743 e-06$ & $5.6460 e-08$ & $1.7653 e-06$ & $1.3254 e-07$ & $1.8889 e-09$ \\
\hline \multicolumn{7}{|l|}{$\theta_{1}=0.9$} \\
\hline $\operatorname{Bias}(\alpha)$ & $-4.6077 e-04$ & $-6.1094 e-05$ & $6.5116 e-06$ & $-2.1260 E-04$ & $1.8841 E-05$ & $1.3932 E-06$ \\
\hline $\operatorname{MSE}(\alpha)$ & $6.3694 e-05$ & $1.1197 e-06$ & $1.2720 e-08$ & $1.3559 E-05$ & $1.0649 E-07$ & $5.8233 E-10$ \\
\hline
\end{tabular}

By the (ii), (iv), and (vi) of Theorem 5 , we have

$$
\begin{gathered}
T^{-2} \sum_{t=1}^{T} X_{t-1} u_{t} \Longrightarrow \frac{1}{2} \mu_{\varepsilon}^{2}\left(1+\sum_{k=1}^{q} \theta_{k}\right)^{2}, \\
T^{-2} \sum_{t=1}^{T} X_{t-1} \Longrightarrow \frac{1}{2} \mu_{\varepsilon}\left(1+\sum_{k=1}^{q} \theta_{k}\right), \\
T^{-2} \sum_{t=1}^{T} X_{t}=T^{-2} \sum_{t=1}^{T} X_{t-1}+T^{-1}\left(T^{-1} \sum_{t=1}^{T} u_{t}\right) \\
\Longrightarrow \frac{1}{2} \mu_{\varepsilon}\left(1+\sum_{k=1}^{q} \theta_{k}\right), \\
T^{-3} \sum_{t=1}^{T} X_{t-1}^{2} \Longrightarrow \frac{1}{3} \mu_{\varepsilon}^{2}\left(1+\sum_{k=1}^{q} \theta_{k}\right)^{2} .
\end{gathered}
$$

Then, we get

$$
\begin{gathered}
T^{-4}\left(T \sum_{t=1}^{T} X_{t-1} X_{t}-\left(\sum_{t=1}^{T} X_{t-1}\right)\left(\sum_{t=1}^{T} X_{t}\right)\right) \\
\Longrightarrow \frac{1}{3} \mu_{\varepsilon}^{2}\left(1+\sum_{k=1}^{q} \theta_{k}\right)^{2}+0-\left(\frac{1}{2} \mu_{\varepsilon}\left(1+\sum_{k=1}^{q} \theta_{k}\right)\right)^{2} \\
=\frac{1}{12} \mu_{\varepsilon}^{2}\left(1+\sum_{k=1}^{q} \theta_{k}\right)^{2}
\end{gathered}
$$

Similarly, we have the denominator limit of (5):

$$
\begin{gathered}
T^{-4}\left(T \sum_{t=1}^{T} X_{t-1}^{2}-\left(\sum_{t=1}^{T} X_{t-1}\right)^{2}\right) \\
\Longrightarrow \frac{1}{12} \mu_{\varepsilon}^{2}\left(1+\sum_{k=1}^{q} \theta_{k}\right)^{2}
\end{gathered}
$$

Using the Slutsky theorem, we obtain

$$
\begin{aligned}
\widehat{\alpha} & =\frac{T \sum_{t=1}^{T} X_{t-1} X_{t}-\left(\sum_{t=1}^{T} X_{t-1}\right)\left(\sum_{t=1}^{T} X_{t}\right)}{T \sum_{t=1}^{T} X_{t-1}^{2}-\left(\sum_{t=1}^{T} X_{t-1}\right)^{2}} \\
& =\frac{T^{-4}\left(T \sum_{t=1}^{T} X_{t-1} X_{t}-\left(\sum_{t=1}^{T} X_{t-1}\right)\left(\sum_{t=1}^{T} X_{t}\right)\right)}{T^{-4}\left(T \sum_{t=1}^{T} X_{t-1}^{2}-\left(\sum_{t=1}^{T} X_{t-1}\right)^{2}\right)} \\
& \Longrightarrow \frac{(1 / 12) \mu_{\varepsilon}^{2}\left(1+\sum_{k=1}^{q} \theta_{k}\right)^{2}}{(1 / 12) \mu_{\varepsilon}^{2}\left(1+\sum_{k=1}^{q} \theta_{k}\right)^{2}}=1 .
\end{aligned}
$$

This completes the proof.

\section{Simulation Study}

To study the empirical performance of the CLS estimator of the autoregressive coefficient for an auxiliary regression process, while the true process is $\operatorname{NSINARMA}(1, q)$ process, a brief simulation study is conducted.

Consider the true process,

$$
\begin{gathered}
X_{t}=X_{t-1}+u_{t}, \\
u_{t}=\varepsilon_{t}+\theta_{1} \circ \varepsilon_{t-1}+\cdots+\theta_{q} \circ \varepsilon_{t-q},
\end{gathered}
$$


TABLE 2: Bias and MSE results of $\alpha$ for NSINARMA(1,2) model.

\begin{tabular}{|c|c|c|c|c|c|c|}
\hline \multicolumn{7}{|c|}{ CLS } \\
\hline & \multicolumn{3}{|c|}{$\lambda=0.3$} & \multicolumn{3}{|c|}{$\lambda=5$} \\
\hline Sample size & 100 & 300 & 800 & 100 & 300 & 800 \\
\hline \multicolumn{7}{|c|}{$\left(\theta_{1}, \theta_{2}\right)=(0.1,0.1)$} \\
\hline $\operatorname{Bias}(\alpha)$ & $-9.8012 e-04$ & $-8.0314 e-05$ & $-1.2149 e-05$ & $1.6030 e-05$ & $-2.6319 e-05$ & $-7.9892 e-06$ \\
\hline $\operatorname{MSE}(\alpha)$ & $2.8819 e-04$ & $1.9351 e-06$ & $4.4280 e-08$ & $7.7085 e-07$ & $2.0781 e-07$ & $1.9148 e-08$ \\
\hline \multicolumn{7}{|c|}{$\left(\theta_{1}, \theta_{2}\right)=(0.1,0.6)$} \\
\hline $\operatorname{Bias}(\alpha)$ & $-9.0876 e-04$ & $-1.4515 e-04$ & $3.6936 e-06$ & $-4.5047 e-05$ & $-3.6633 e-05$ & $-1.7588 e-06$ \\
\hline $\operatorname{MSE}(\alpha)$ & $2.4775 e-04$ & $6.3203 e-06$ & $4.0928 e-09$ & $6.0877 e-07$ & $4.0259 e-07$ & $9.2803 e-10$ \\
\hline \multicolumn{7}{|c|}{$\left(\theta_{1}, \theta_{2}\right)=(0.2,0.3)$} \\
\hline $\operatorname{Bias}(\alpha)$ & $-5.8582 e-04$ & $-8.2571 e-05$ & $-1.1720 e-05$ & $4.7792 e-05$ & $-2.2042 e-05$ & $-7.8901 e-06$ \\
\hline $\operatorname{MSE}(\alpha)$ & $1.0296 e-04$ & $2.0454 e-06$ & $4.1208 e-08$ & $6.8522 e-07$ & $1.4575 e-07$ & $1.8676 e-08$ \\
\hline \multicolumn{7}{|c|}{$\left(\theta_{1}, \theta_{2}\right)=(0.3,0.4)$} \\
\hline $\operatorname{Bias}(\alpha)$ & $-1.0548 e-03$ & $-1.3550 e-04$ & $-2.1872 e-06$ & $-6.0986 e-05$ & $-2.1873 e-05$ & $-1.6658 e-08$ \\
\hline $\operatorname{MSE}(\alpha)$ & $3.3379 e-04$ & $5.5082 e-06$ & $1.4352 e-09$ & $1.1158 e-06$ & $1.4353 e-07$ & $8.3243 e-14$ \\
\hline \multicolumn{7}{|c|}{$\left(\theta_{1}, \theta_{2}\right)=(0.4,0.4)$} \\
\hline $\operatorname{Bias}(\alpha)$ & $-8.9921 e-04$ & $-1.4099 e-04$ & $-8.5553 e-07$ & $-6.6568 e-05$ & $-2.2816 e-05$ & $1.0669 e-07$ \\
\hline $\operatorname{MSE}(\alpha)$ & $2.4257 e-04$ & $5.9633 e-06$ & $2.1958 e-10$ & $1.3294 e-06$ & $1.5617 e-07$ & $3.4148 e-12$ \\
\hline
\end{tabular}

TABLE 3: Bias and MSE results of $\alpha$ for NSINARMA(1,3) model.

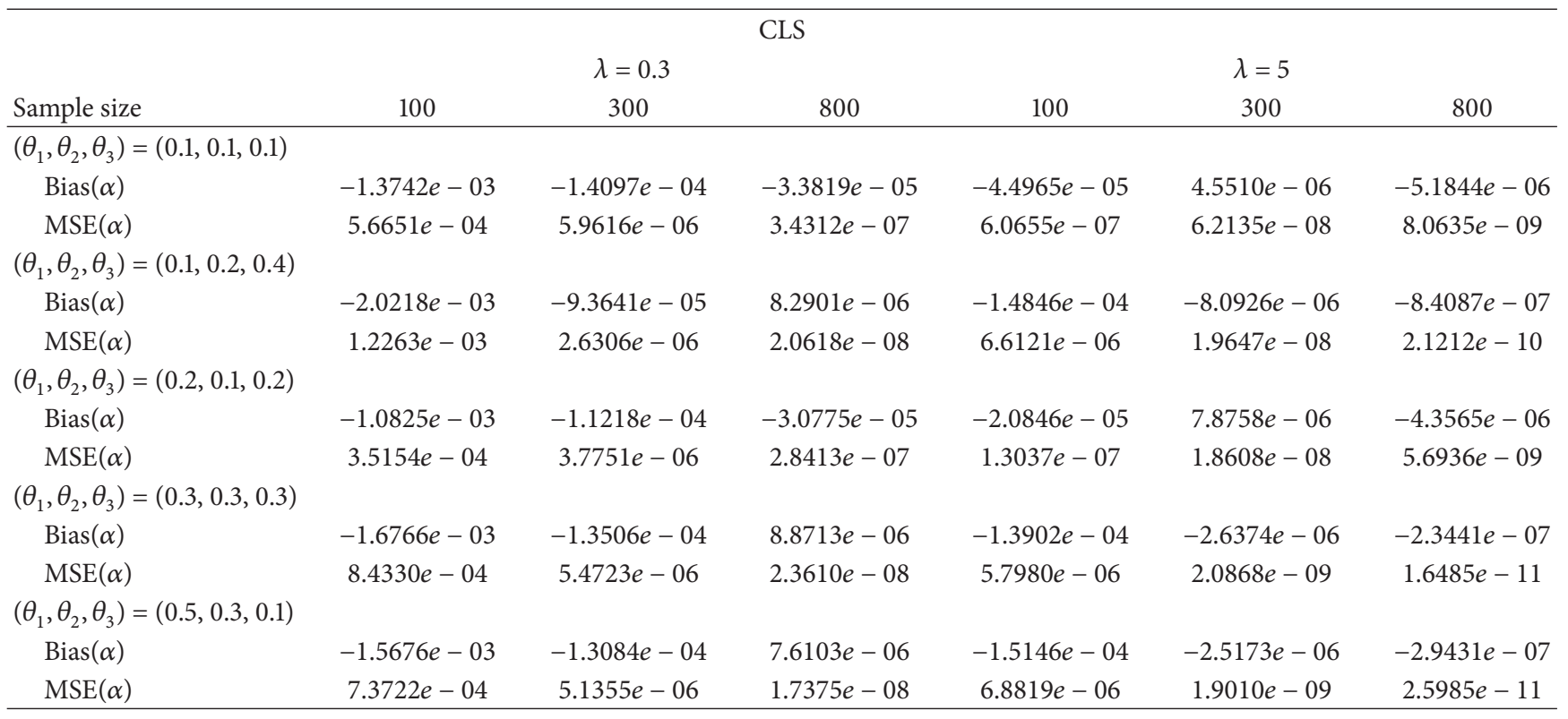

where $\left\{\varepsilon_{t}\right\}$ is an an i.i.d. sequence of the Poisson random variables with parameter $\lambda=0.3,5$. The lag orders and coefficient parameters values considered are

(i) for $q=1, \theta_{1} \in\{0.1,0.4,0.7,0.9\}$,

(ii) for $q=2,\left(\theta_{1}, \theta_{2}\right) \in\{(0.1,0.1),(0.2,0.3),(0.1,0.6)$, $(0.3,0.4),(0.4,0.4)\}$

(iii) for $q=3,\left(\theta_{1}, \theta_{2}, \theta_{3}\right) \in\{(0.1,0.1,0.1),(0.1,0.2,0.4)$, $(0.2,0.1,0.2),(0.3,0.3,0.3),(0.5,0.3,0.1)\}$.

The auxiliary regression process is an INAR(1) process,

$$
X_{t}=\alpha \circ X_{t-1}+v_{t} \text {, }
$$

where $\left\{v_{t}\right\}$ is a sequence of i.i.d. nonnegative integer-valued random variables. This simulation study is conducted to indicate the large sample performances of the CLS estimator of $\alpha$ when the true process is $\operatorname{NSINARMA}(1, q)$ process. In the simulation, we use $X_{0}=\varepsilon_{0}=\varepsilon_{-1}=\cdots=\varepsilon_{-q}=0$. The study is based on 300 replications. For each replication, we estimate the model parameter $\alpha$ and calculate the bias and MSE of the parameter estimates. The sample size is varied to be $T=100,300$, and 800 .

From the results reported in Tables 1, 2, and 3, we can see that CLS is a good estimation method. The estimates' bias and MSE values are all small. Most of the biases are negative. 
All the bias and MSE values decrease with an increasing sample size $T$. When the coefficient $\theta$ of innovation process and the sample size are fixed, we find that the absolute values of bias and MSE become smaller with an increasing $\lambda$. When the sample size is increased, the MSE and bias values both converge to zero. For example, the smallest bias and MSE values in the simulation showed in Table 1 are $9.7185 e-07$ and $2.8335 e-10$. This illustrates that the CLS estimator $\widehat{\alpha}$ given by (5) converges to a constant.

\section{Conclusions}

In this paper, we have proposed a nonstationary INAR model for nonstationary integer-valued data. We have used an extended structure of the innovation process to allow the innovation with correlation to follow an $\operatorname{INMA}(q)$ process. We have presented the moments and conditional moments for this model, proposed a CLS estimator for the autoregressive coefficient in auxiliary regression model, and obtained the asymptotic distribution for the estimator of coefficient. The simulation results indicate that our CLS method produces good estimates for large samples.

\section{Acknowledgments}

The authors are grateful to two anonymous referees for helpful comments and suggestions which greatly improved the paper. This work is supported by the National Natural Science Foundation of China (no. 71171166 and no. 71201126), the Science Foundation of Ministry of Education of China (no. 12XJC910001), and the Fundamental Research Funds for the Central Universities (no. JBK120405).

\section{References}

[1] N. Silva, I. Pereira, and M. E. Silva, "Forecasting in $\operatorname{INAR(1)}$ model," REVSTAT Statistical Journal, vol. 7, no. 1, pp. 119-134, 2009.

[2] C. H. Weiß, "Thinning operations for modeling time series of counts-a survey," Advances in Statistical Analysis, vol. 92, no. 3, pp. 319-341, 2008.

[3] R. Ferland, A. Latour, and D. Oraichi, "Integer-valued GARCH process," Journal of Time Series Analysis, vol. 27, no. 6, pp. 923942, 2006.

[4] K. Fokianos and R. Fried, "Interventions in INGARCH processes," Journal of Time Series Analysis, vol. 31, no. 3, pp. 210-225, 2010.

[5] C. H. Weiß, "The INARCH(1) model for overdispersed time series of counts," Communications in Statistics-Simulation and Computation, vol. 39, no. 6, pp. 1269-1291, 2010.

[6] F. Zhu, "A negative binomial integer-valued GARCH model," Journal of Time Series Analysis, vol. 32, no. 1, pp. 54-67, 2011.

[7] F. Zhu, "Modeling overdispersed or underdispersed count data with generalized Poisson integer-valued GARCH models," Journal of Mathematical Analysis and Applications, vol. 389, no. 1, pp. 58-71, 2012.

[8] F. Zhu and D. Wang, "Diagnostic checking integer-valued $A R C H(p)$ models using conditional residual autocorrelations,"
Computational Statistics \& Data Analysis, vol. 54, no. 2, pp. 496508, 2010.

[9] R. Bu and B. McCabe, "Model selection, estimation and forecasting in $\operatorname{INAR}(p)$ models: a likelihood-based Markov Chain approach," International Journal of Forecasting, vol. 24, no. 1, pp. 151-162, 2008.

[10] V. Enciso-Mora, P. Neal, and T. Subba Rao, "Efficient order selection algorithms for integer-valued ARMA processes," Journal of Time Series Analysis, vol. 30, no. 1, pp. 1-18, 2009.

[11] B. P. M. McCabe, G. M. Martin, and D. Harris, "Efficient probabilistic forecasts for counts," Journal of the Royal Statistical Society B, vol. 73, no. 2, pp. 253-272, 2011.

[12] H. Zheng, I. V. Basawa, and S. Datta, "Inference for pth-order random coefficient integer-valued autoregressive processes," Journal of Time Series Analysis, vol. 27, no. 3, pp. 411-440, 2006.

[13] H. Zheng, I. V. Basawa, and S. Datta, "First-order random coefficient integer-valued autoregressive processes," Journal of Statistical Planning and Inference, vol. 137, no. 1, pp. 212-229, 2007.

[14] D. Gomes and L. C. e Castro, "Generalized integer-valued random coefficient for a first order structure autoregressive (RCINAR) process," Journal of Statistical Planning and Inference, vol. 139, no. 12, pp. 4088-4097, 2009.

[15] K. Yu, D. Shi, and P. Song, "First-order random coefficient integer-valued moving average process," Journal of Zhejiang University-Science Edition, vol. 37, no. 2, pp. 153-159, 2010.

[16] K. Yu, D. Shi, and H. Zou, "The random coefficient discretevalued time series model," Statistical Research, vol. 28, no. 4, pp. 106-112, 2011 (Chinese).

[17] M. Kachour and J. F. Yao, "First-order rounded integer-valued autoregressive $(\operatorname{RINAR(1))}$ process," Journal of Time Series Analysis, vol. 30, no. 4, pp. 417-448, 2009.

[18] H.-Y. Kim and Y. Park, "A non-stationary integer-valued autoregressive model," Statistical Papers, vol. 49, no. 3, pp. 485-502, 2008.

[19] H. Zhang, D. Wang, and F. Zhu, "Inference for $\operatorname{INAR}(p)$ processes with signed generalized power series thinning operator," Journal of Statistical Planning and Inference, vol. 140, no. 3, pp. 667-683, 2010.

[20] M. Kachour and L. Truquet, "A p-order signed integer-valued autoregressive (SINAR( $p))$ model," Journal of Time Series Analysis, vol. 32, no. 3, pp. 223-236, 2011.

[21] J. Hellström, "Unit root testing in integer-valued AR(1) models," Economics Letters, vol. 70, no. 1, pp. 9-14, 2001.

[22] M. Ispány, G. Pap, and M. C. A. van Zuijlen, "Asymptotic inference for nearly unstable INAR(1) models," Journal of Applied Probability, vol. 40, no. 3, pp. 750-765, 2003.

[23] L. Györfi, M. Ispány, G. Pap, and K. Varga, "Poisson limit of an inhomogeneous nearly critical INAR(1) model," Acta Universitatis Szegediensis, vol. 73, no. 3-4, pp. 789-815, 2007.

[24] F. C. Drost, R. van den Akker, and B. J. M. Werker, "The asymptotic structure of nearly unstable non-negative integervalued AR(1) models," Bernoulli, vol. 15, no. 2, pp. 297-324, 2009.

[25] M. Barczy, M. Ispány, and G. Pap, "Asymptotic behavior of unstable $\operatorname{INAR}(p)$ processes," Stochastic Processes and their Applications, vol. 121, no. 3, pp. 583-608, 2011.

[26] J. D. Hamilton, Time Series Analysis, Princeton University Press, Princeton, NJ, USA, 1994. 


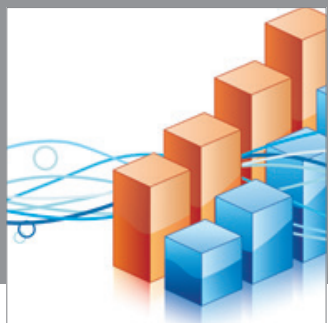

Advances in

Operations Research

mansans

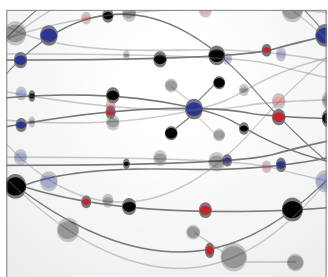

The Scientific World Journal
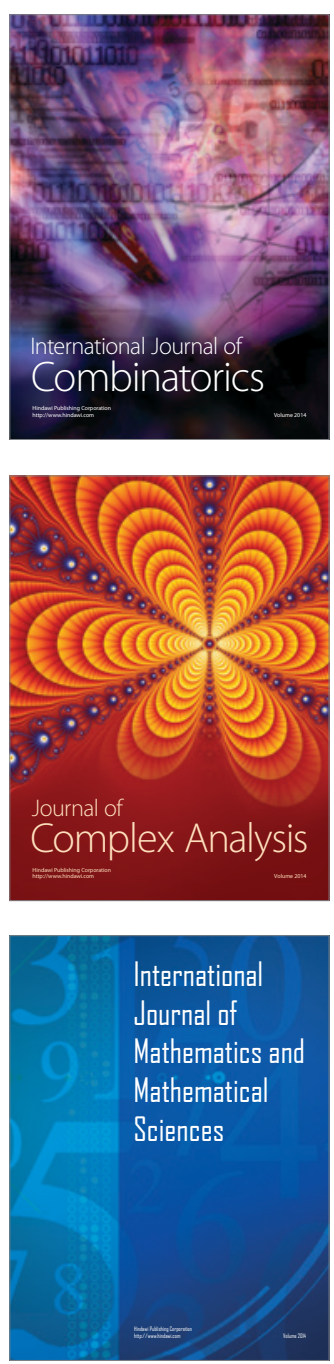
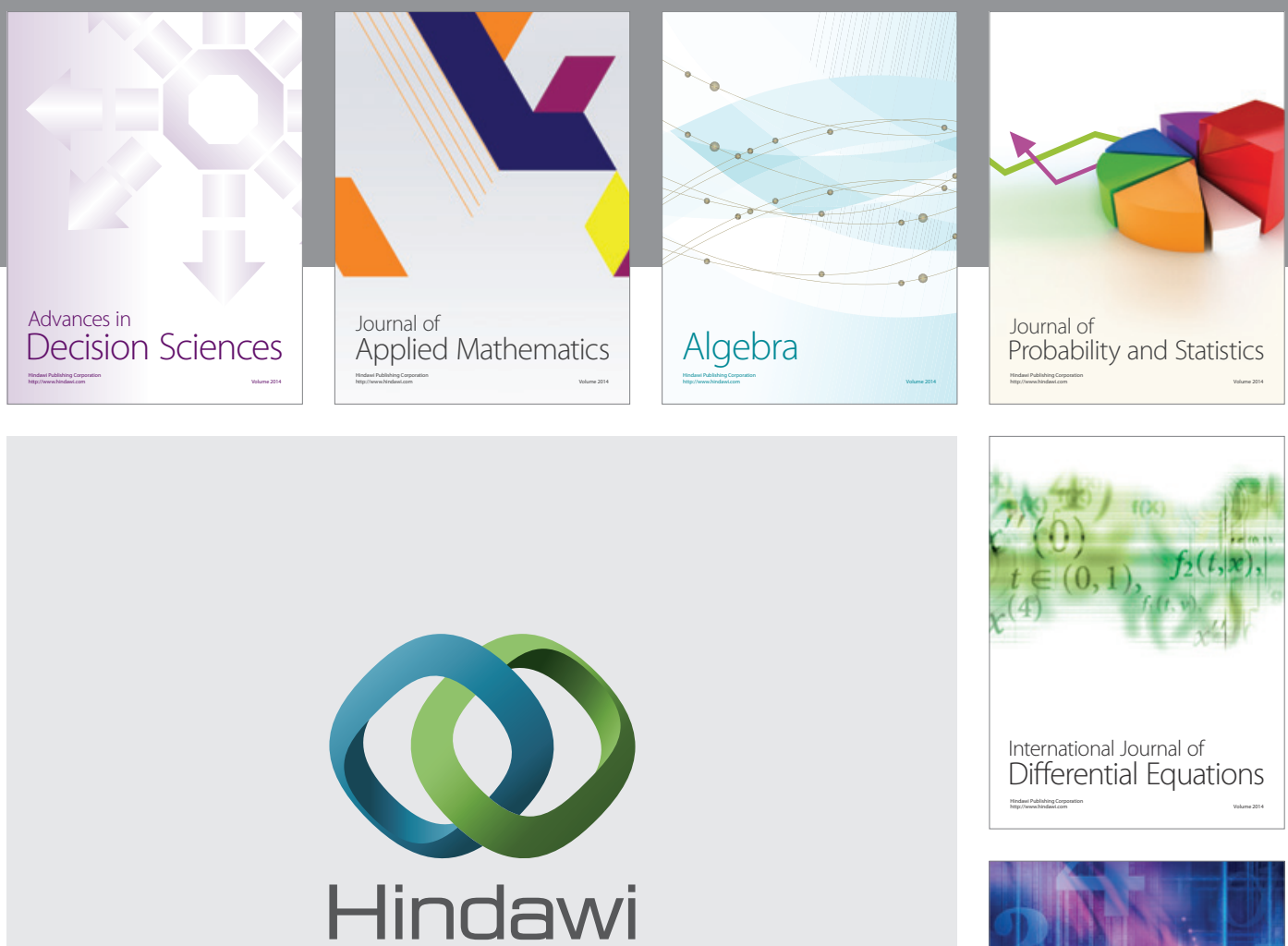

Submit your manuscripts at http://www.hindawi.com
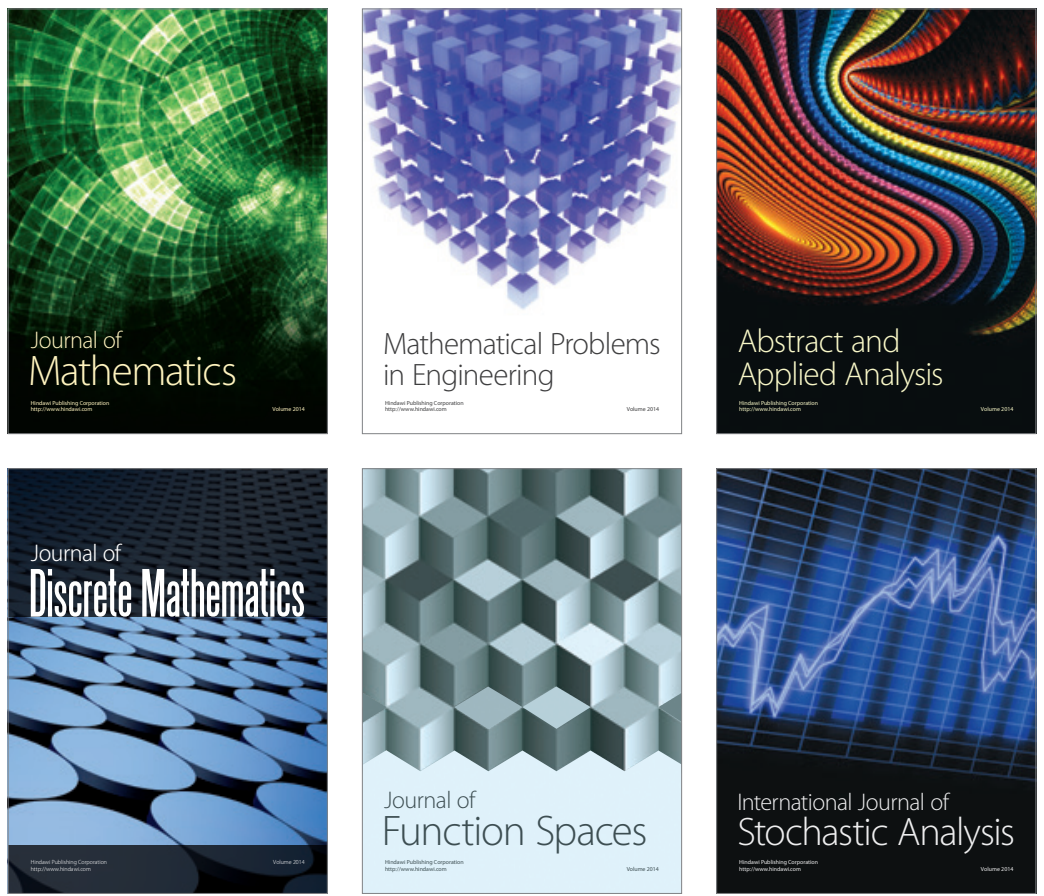

Journal of

Function Spaces

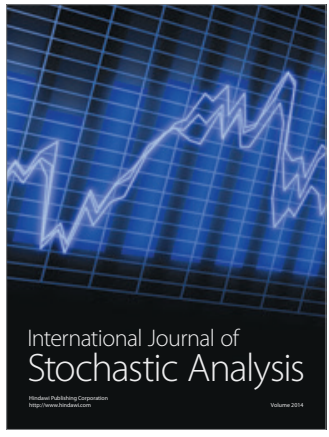

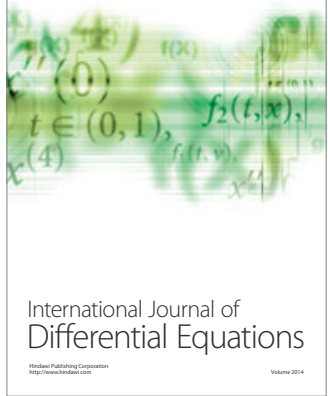
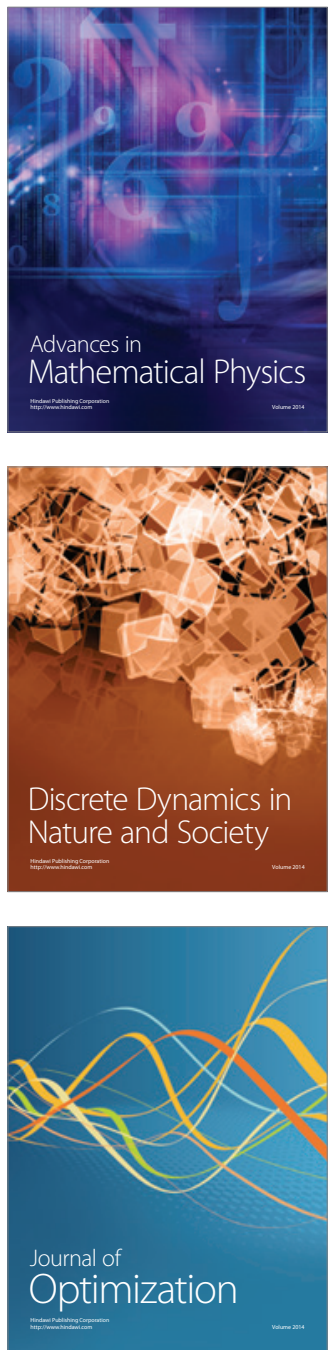